\title{
Suicide by self-poisoning with pesticides: the need for action
}

\author{
José M Bertolote* and Alexandra Fleischmann* \\ * Management of Mental and Brain Disorders, Department of Mental Health and Substance Abuse, World Health Organization, Geneva, Switzerland
}

\begin{abstract}
Self-poisoning with pesticides is a serious public health problem in many agricultural communities of lowand middle-income countries around the world. Pesticides are readily available in most rural households and commonly used in acts of intentional self-poisoning in moments of crisis. Due to the very high case fatality of highly toxic pesticides many impulsive acts are turned into deaths (Mishara, 2007; Phillips et al., 2002). As pesticide ingestion is acknowledged as one of the most frequently used methods of suicide worldwide (Bertolote et al., 2006a; Eddleston et al., 2006; Gunnell and Eddleston, 2003) strategies to reduce self-poisoning with pesticides should have a major impact on the global burden of suicide (Bertolote et al., 2006b).
\end{abstract}

\section{The burden}

Based on data from Sri Lanka, Jeyaratnam estimated already in 1985 that approximately 220,000 deaths every year occurred from pesticide poisoning in the developing world (Jeyaratnam, 1985). Almost 20 years later, it was estimated that there were around 300,000 suicides from intentional ingestions of pesticides every year worldwide, coming from rural Asia in particular (Gunnell and Eddleston, 2003). This estimate was based on the research evidence which suggested that pesticide ingestion accounted for over $60 \%$ of suicides in many rural areas of China and South-East Asia.

The most recent estimate of deaths from pesticide poisoning used data from all regions of the World Health Organization (WHO) for the first time.

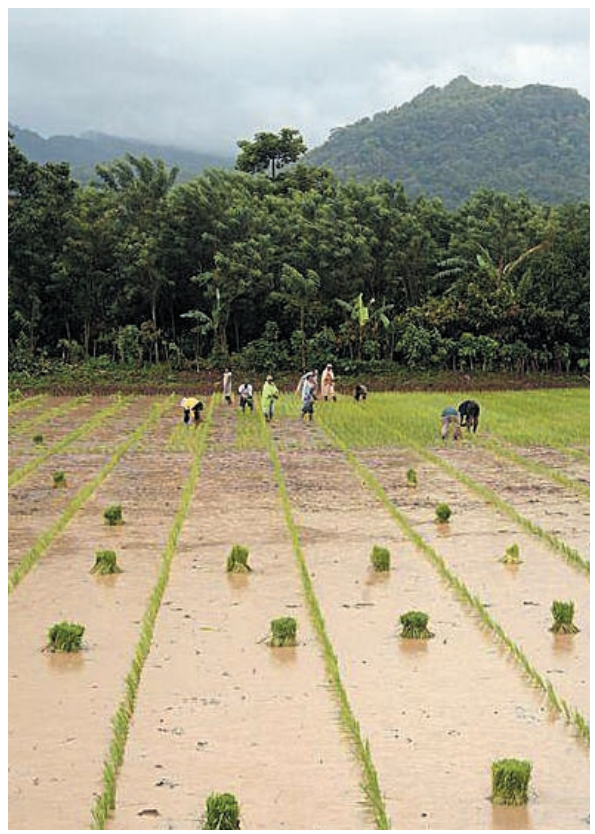

As a conservative estimate 258,234 was reported as the annual number of pesticide suicides worldwide, with a plausible range of 234,000 to 326,000 (Gunnell et al., 2007). This accounts for approximately one third of all suicides globally. Since the reliability of suicide statistics for India were questioned and taking into account recent studies of rural India, the authors suggested that the estimated number of suicides due to pesticides could even be as high as 371,594.

In that same paper, Gunnell et al. (2007) presented a systematic review of studies of fatal pesticide self-poisoning around the world; among those were the following: In China and Malaysia, both countries of the WHO Western Pacific Region, studies reported that $62 \%$ and over $90 \%$ of suicides, respectively, were the result of selfpoisoning with pesticides. In the WHO South-East Asia Region, a study from Sri Lanka showed the highest proportion of suicide by pesticide ingestion $(70 \%)$. Data from countries in Central and South America of the WHO Region of the Americas also testified of the importance of pesticides in suicide, e.g. Trinidad (68\%) and Suriname $(50 \%)$. This can be complemented by information from the WHO Mortality Database where self-poisoning with pesticides was employed in $86 \%$ of the suicides in El Salvador. In the WHO African Region, a report from Malawi had the highest proportion of pesticides in suicide $(79 \%)$. In Pakistan, a country of the WHO
Eastern Mediterranean Region, 39\% pesticide suicides were reported in one study. In the WHO European Region, suicides by pesticide ingestion were reported from some areas, such as the Almeria province in Spain (22\%), and the South-West of Greece (19\%).

\section{Taking global action}

In view of pesticide self-poisoning as a major public health problem and the relative little attention it has received so far in comparison with the magnitude of its burden (both in terms of mortality and morbidity), WHO launched an intersectoral public health initiative entitled "The Impact of Pesticides on Health: Preventing Intentional and Unintentional Deaths from Pesticide Poisoning" as part of the WHO worldwide initiative for the prevention of suicide (SUPRE). As they share a common interest and concern, the WHO Department of Mental Health and Substance Abuse, the Department of Violence, Injury Prevention and Disability, and the Programme on the Promotion of Chemical Safety joined forces and work together with other partners, both within and outside the organization, to advance this important initiative.

The overall goal is to reduce mortality and morbidity related to pesticide poisoning. More specifically, the objectives are as follows:

i) review and recommend improved pesticide regulatory policies, 
ii) implement sustainable epidemiological surveillance and monitoring of pesticide poisoning in clinical settings and communities,

iii) improve the medical management and mental health care of people with pesticide poisoning in health care facilities at different levels,

iv) provide training in the safe handling of pesticides and the identification and management of pesticide poisoning at different sectors and levels, and

v) develop or strengthen community programmes that minimize risks of intentional and unintentional pesticide poisoning.

When non-fatal and fatal self-poisoning by pesticides plays an important role in suicidal behaviour, it is key that relevant preventive measures are anchored in national suicide prevention programmes; these, unfortunately, mostly do not exist as yet in low- and middle income countries. To this end, WHO has started to work with governments worldwide to raise awareness of the importance and magnitude of suicidal behaviours and to provide technical assistance in the development and implementation of national strategies for suicide prevention.

It is crucial that governments ratify, implement and enforce the Hazardous Chemicals and Wastes Conventions, i.e. the Basel, Rotterdam, and Stockholm Conventions, which cover key elements of the management of hazardous chemicals (www.basel.int; www.pic.int; www.pops.int; last accessed on 10 January 2007). Whereas this first crucial step has been done by many countries, the implementation poses major challenges due to barriers, such as the shortage of funds, the lack of expertise, human resources and training, the need of advanced technologies and equipment, the limited database systems available, and the lack of public awareness. Konradsen et al (2005) stressed the need of these conventions not only to propose in the implementation actions such as phasing out the most toxic chemicals, promoting alternative pest management strategies, or improving safe storage, but also to explicitly include self-poisonings in risk assessments and to directly deal with the subject of self-harm.

Regarding the third objective (iii) of the above-mentioned WHO initiative, a WHO meeting of experts was convened in December 2007 to identify effective clinical management of acute pesticide intoxication (both accidental and intentional) at different levels in the health care system, i.e. primary health care, district hospitals, and specialized units. Although there had been several comprehensive or limited guidelines on this clinical management, some of which produced by different programmes in $\mathrm{WHO}$, time had come to do an in-depth review of these previous initiatives and translate them into clear clinical guidance to be used by health staff, indicating its precise insertion within the health care system.

Once this has been made available, training, as indicated in objective (iv) above, can be initiated at the different levels. Also, in view of the scarcity of information, another major objective (ii) is to implement sustainable

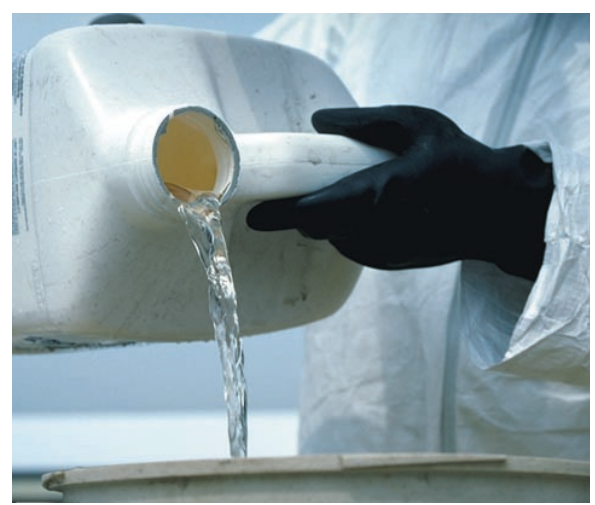

epidemiological surveillance and monitoring of pesticide poisoning in clinical settings and communities.

\section{Collaboration with the International Association for Suicide Prevention (IASP)}

As to the fifth objective (v), WHO and IASP, an international nongovernmental organization in official relations with WHO, have agreed on a project collaboration to conduct jointly activities related to "Best practices on community action for safer access to pesticides". This collaboration began with a meeting of experts in 2006 to identify the current state of knowledge on effective and acceptable community interventions that have significant potential in preventing self-harm by pesticide poisoning. The basic information and recommendations arising from that meeting were summarized in the publication entitled "Safer Access to Pesticides: Community Interventions" (World Health

Organization, 2006).

Among the community interventions identified were those addressing safer storage, education, and psychosocial interventions, as follows:

- Locked boxes for storing pesticides in farming households;

- Centralized communal storage of pesticides;

- Training of pesticide users about health risks associated with pesticide use and about safe use, storage and disposal of pesticides;

- Training of resource persons/ opinion leaders to ensure that they have the most up-to-date information on the prevention, identification and acute management of health problems associated with pesticide use;

- Training of retailers to teach pesticide users about health risks and appropriate use, storage and 
disposal of pesticides and to monitor their compliance with regulations;

- Encouragement of the local media to support programmes aimed at reducing pesticide-related suicides and to decrease inappropriate reporting of suicides that can lead to copycat suicides; and

- Training of school children about sage use, storage and disposal of pesticides, and psychosocial interventions to augment communitybased pesticide management measures.

The following recommendations were formulated:

- Countries review the list of authorized agricultural products and eliminate those that do not meet the Basel, Rotterdam, and Stockholm conventions;

- Countries explore the impact of fatal and non-fatal suicidal behaviour in the national mortality profile and national injury profile and the specific role of pesticides therein; and

- In countries or regions where intentional and unintentional pesticide poisoning represents a public health problem, interested parties (governments, regions, communities, NGOs, etc.) should initiate a series of specific steps to assess the feasibility and effectiveness of the proposed community interventions, and adapt them to local conditions, implement them and evaluate the outcomes and their sustainability.
Subsequently, in December 2007, in collaboration with IASP, a meeting on "Feasibility Demonstration Projects on Community Interventions for Safer Access to Pesticides" was held to select interventions from those that had been identified previously and to prepare protocols to conduct feasibility demonstration projects related to these actions. The expected outcomes of the meeting were protocols for the demonstration of feasibility of community-level interventions for safer access to pesticides and the identification of sites, where to conduct those demonstration projects, and their principal investigators. Eligible proposals included centralized communal storage of pesticides, cooperative purchase and communal distribution of pesticides at village level, secure storage of pesticides, and community-wide educational and promotional efforts to raise awareness about the health risks associated with pesticide use.

\section{Conclusion}

The success of the intersectoral public health initiative to reduce the mortality and morbidity related to pesticide poisoning will depend on broad intersectoral and financial support, involving the governmental and private sector, which includes relevant nongovernmental organizations, and agencies of the United Nations.

\section{References}

Bertolote JM, Fleischmann A, Butchart A, Besbelli N. Suicide, suicide attempts and pesticides: a major hidden public health problem. Bulletin of the World Health Organization 2006a; 84, 260-261.

Bertolote JM, Fleischmann A, Eddleston M, Gunnell, D. Deaths from pesticide poisoning: a global response. British Journal of Psychiatry 2006b; 189, 201-203.

Eddleston M, Buckley NA, Gunnell D, Dawson $\mathrm{AH}$, Konradsen F. Identification of strategies to prevent death after pesticide self-poisoning using a Haddon matrix. Injury Prevention 2006; $12,333-337$.
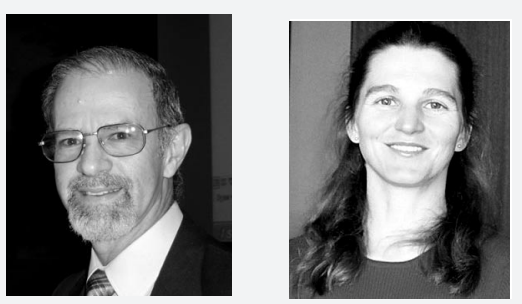

José Manoel Bertolote is Coordinator of the Team on Management of Mental and Brain Disorders, Department of Mental Health and Substance Dependence, World Health Organization, Geneva, Switzerland. One of his responsibilities is SUPRE, the WHO Global Initiative on Suicide Prevention. He is also Associate Professor in the Department of Psychogeriatrics at Lausanne University, Switzerland.

Alexandra Fleischmann works in the Team of Management of Mental and Brain Disorders, Department of Mental Health and Substance Abuse, World Health Organization, Geneva, Switzerland.

The authors are staff members of the World Health Organization. The authors alone are responsible for the views expressed in this publication and they do not necessarily represent the decisions, policy or views of the World Health Organization.

Gunnell D, Eddleston M, Phillips MR, Konradsen F. The global distribution of fatal pesticide self-poisoning: systematic review. BMC Public Health 2007; 7, 357-395.

Gunnell D, Eddleston M. Suicide by intentional ingestion of pesticides: a continuing tragedy in developing countries. International Journal of Epidemiology 2003; 32, 902-909.

Jeyaratnam J. Health problems of pesticide usage in the third world. British Journal of Industrial Medicine 1985; 42, 505-506.

Konradsen F, van der Hoek W, Gunnell D, Eddleston, M. Missing deaths from pesticide self-poisoning in the IFCS Forum IV. Bulletin of the World Health Organization 2005; 83, 157-158.

Mishara BL. Prevention of deaths from intentional pesticide poisoning. Crisis 2007; 28 (Suppl.1), 10-20.

Phillips MR, Yang G, Zhang Y, Wang L, Ji H, Zhou M. Risk factors for suicide in China: a national case-control psychological autopsy study. Lancet 2002; 360, 1728-1736.

World Health Organization. Safer access to pesticides: Community interventions. Geneva: WHO, 2006. 